\title{
Integrative Analysis of Systems Pharmacology and Proteomics to Identify Key Targets and Pathways of Tuomin-Zhiti-Decoction for Allergic Rhinitis
}

\section{Jinjun Cheng}

Beijing University of Chinese Medicine

Meiling Zhang

Beijing University of Chinese Medicine

Yanfei Zheng

Beijing University of Chinese Medicine

Ji Wang

Beijing University of Chinese Medicine

Qi Wang ( $\nabla$ wangqi710@126.com )

Beijing University of Chinese Medicine

\section{Research}

Keywords: Tuomin-Zhiti-Decoction, Allergic rhinitis, Chinese herbal formula, systems pharmacology, proteomics, Molecular docking

Posted Date: November 23rd, 2021

DOI: https://doi.org/10.21203/rs.3.rs-1068059/v1

License: (c) (i) This work is licensed under a Creative Commons Attribution 4.0 International License. Read Full License 


\section{Abstract}

Background: Tuomin-Zhiti-Decoction (TZD) is a polyherbal medicine consisting of eleven different herbs and has a significant effect on Allergic rhinitis (AR), yet its underlying mechanism is still unknown.

Methods: Mice were sensitized and intranasally challenged with ovalbumin (OVA), the nasal symptoms, Th1/Th2-related cytokines and histopathology were examinated after TZD treatments. Bio-active compounds, potential therapeutic targets and underlying mechanisms of TZD against AR were systematically elucidated by integrating systems pharmacology approach and proteomics analysis. Then we validated the binding affinity between key targets and their corresponding active compounds using molecular docking evaluation.

Results: A total of 280 active ingredients, 240 targets of TZD and 643 AR-related targets were obtained. Furthermore, 74 overlapping targets were identified. TZD oral administrations inhibited allergic responses via reduction of OVA-specific immunoglobulin $E(\mathrm{IgE})$ levels and histamine release. In nasal tissue, TZD not only decreased nasal rubbing and sneezing to AR mice, but also reduced AR-induced damage to nasal mucosa, accordingly, the nasal symptoms were also clearly ameliorated. Moreover, TZD modulated the balance of Th1/Th2/Th17. The proteomics analysis identified 41 differentially expressed proteins (DEPs), protein function analysis showed that the DEPs were mainly involved in PI3K-AKT, MAPK, EGFR tyrosine kinase inhibitor resistance, NF-kappa B signaling pathway. Our results indicate that TZD plays important roles by restoring DEPs, through the selection of key DEPs, IL-6 and CD40 may be potential protein targets of TZD in treatment of AR. Combining the systems pharmacology, quercetin and wogonin may play more effective roles in AR. Molecular docking confirmed that most active compounds of TZD could bind tightly to the key targets.

Conclusion: TZD may have therapeutic potential for treating AR, integrating analysis of systems pharmacology and proteomics uncovered the underlying mechanism and targets of TZD, which provides a scientific method for the rational development of traditional Chinese medicine.

\section{Introduction}

Allergic rhinitis is one of the most common conditions seen by otolaryngologists, which is a type I allergic disease of the nasal mucosa, characterized by paroxysmal repetitive sneezing, watery rhinorrhea, and nasal blockage. Over 500 million people suffer from AR since $2010^{1}$ and the prevalence of AR is increasing worldwide. Study of epidemiology of allergic rhinitis shows that in the United States and Europe the lifetime prevalence of AR can be estimated has been estimated to be approximately $2-25 \%$ in children and $1 \%$ to greater than $40 \%$ in adults ${ }^{2,3}$.

AR might not appear to be serious but the burden and costs are substantial ${ }^{4}$. AR reduces the quality of life, impairing sleep quality and cognitive function and causing irritability and fatigue. Annual direct medical costs of AR are substantial, but indirect costs associated with lost work productivity are greater 
than those incurred by asthma ${ }^{3}$. Allergen immunotherapy (AIT) is a proven therapeutic option for the treatment of allergic rhinitis and/or asthma. However, there are no currently available validated biomarkers that can predict AIT success and in some cases, despite appropriate pharmacotherapy and adherence, continue to exhibit exacerbations that appear to be related to allergen exposure. It is requiring the development of new drug.

During recent decades, Chinese herbal medicine, one of the traditional Chinese medicine that serves as a complementary and alternative medicine, has been used as popular strategies for treating AR and aroused a remarkable and growing interest ${ }^{5-7}$. Tuomin-Zhiti-Decoction (TZD), which was optimized based on Chinese herbal formula discovered by well-known professor Qi Wang, is a novel formula of Chinese medicine that has been used to effectively treat AR during recent years.

TZD consists of eleven Chinese herbs, including Glycyrrhizae Radix et Rhizoma, Magnoliae Flos, Xanthii Fructus, Ganoderma, Lilii Bulbus, Scutellariae Radix, Saposhnikoviae Radix, Astragali Radix, Cicadae Periostracum, Mume Fructus, Nidus Vespae. However, TZD's effect on AR has not yet been elucidated. In this study, we evaluated the effect of TZD on allergic responses in ovalbumin-induced allergic rhinitis mice.

\section{Materials And Methods \\ 2.1 Preparation of TZD}

Herbs used in TZD preparation were purchased from the GMP pharmaceutical factory of Beijing Tong Ren Tang Chinese Medicine Co., Ltd.(Beijing, China). TZD used in this study contains eleven different herbal medicines: Glycyrrhizae Radix et Rhizoma (Gancao, GC) 6g, Magnoliae Flos (Xinyi, XY) 10g, Xanthii Fructus (Cangerzi, CEZ) 6g, Ganoderma (Lingzhi, LZ) 10g, Lilii Bulbus (Baihe, BH) 20g, Scutellariae Radix (Huangqin, HQN) 10g, Saposhnikoviae Radix (Fangfeng, FAF) 10g, Astragali Radix (Huangqi, HQI) 30g, Cicadae Periostracum (Chantui, CT) 10g, Mume Fructus (Wumei, WM) 20g, Nidus Vespae (Fengfang, FEF) 10g. TZD was extracted with boiling water for $3 \mathrm{~h}$ and the resulting product was freeze-dried (yield=17.25\%).

\subsection{Network pharmacology analysis}

\subsubsection{Identification of active compounds and prediction of corresponding targets of TZD}

The active constituents of the five herbal medicines in TZD were acquired from the Traditional Chinese Medicine Systems Pharmacology database and Analysis Platform (TCMSP) (https://tcmsp-e.com/) ${ }^{8}$, HERB: a high-throughput experiment- and reference-guided database of traditional Chinese medicine (http://herb.ac.cn/) ${ }^{9}$. According to the recommended drug screening criteria of the TCMSP and HERB database, chemical constituents with oral bioavailability $(O B) \geq 30 \%$ and drug-likeness $(D L) \geq 0.18$ may 
present ideal pharmacological activities, and they were selected as the active ingredients for further analysis. Subsequently, we screened the targets of active ingredients in TZD through the TCMSP database. The target names were imported into the UniProt database (http://www.uniprot.org/) with the species selected as "Homo sapiens," and the gene names of the targets were obtained from the UniProt database ${ }^{10}$.

\subsubsection{Identification of AR-related targets}

"Allergic rhinitis" was used as the keyword to extract the AR-related targets from the Online Mendelian Inheritance in Man (OMIM, https://omim.org/) ${ }^{11}$, DisGeNET database ${ }^{12}$ (DisGeNET, https://www.disgenet.org/), GeneCards (http://www.genecards.org// ${ }^{13}$, and Drugbank (https://www.drugbank.ca/) ${ }^{14}$ databases. The disease-related targets obtained were standardized as gene names from the UniProt database with the species selected as "Homo sapiens." A Venn diagram was drawn online at Venny 2.1 (https://bioinfogp.cnb.csic.es/tools/venny/index.html) to obtain the overlapping targets between the AR-related targets and active compound-related targets, which may be the potential targets of TZD in AR treatment.

\subsubsection{Construction of protein-protein interaction network and screening of hub targets}

The Search Tool for the Retrieval of Interacting Genes (STRING) database (https://string-db.org/) ${ }^{15}$ can explore and analyze the overlapping targets between the AR-related targets and active compound-related targets. In this study, multiple networks were established to visualize and analyze the complicated interconnection of compounds, targets, and disease-related targets using Cytoscape software (https://cytoscape.org/; version 3.8.2). Then the CytoHubba app was used to identify PPI network hub targets $^{16}$. CytoHubba provides eleven calculation methods to identify key proteins in biological networks, and we choose the MCC method for it has a better performance ${ }^{17}$. In these networks, nodes of different colors and shapes represented different active compounds, potential targets, or signal pathways, and the edges represented the connections between the nodes.

\subsection{Animals}

Male Balb/c mice $(20 \pm 2 \mathrm{~g})$ were purchased from Beijing Vital River Laboratory Animal Technology Co., Ltd.. The animals were housed under controlled environmental conditions at a temperature of $22 \pm 3^{\circ} \mathrm{C}$ with a relative humidity of $55 \pm 5 \%$ and $12 \mathrm{~h}$ light/dark cycle throughout the study. This study was performed in accordance with the Guide for the Care and Use of Laboratory Animals and was approved by the Committee of Ethics of Animal Experimentation of the Beijing University of Traditional Chinese Medicine. The mice were divided into six groups $(n=6)$ : group 1, normal saline; group 2, OVA + saline; group 3, OVA+ TZD (6.4 g/kg); group 4, OVA + TZD (3.2 g/kg); group 5, OVA + TZD (1.6 g/ $/ \mathrm{kg})$; group 6, loratadine (LTD) $(1.5 \mathrm{mg} / \mathrm{kg})$.

\subsection{Preparation of OVA-induced allergic rhinitis mouse model}


Male Balb/c mice (6 weeks old) were given free access to a standard laboratory diet and water during the experimental period. OVA solution $\left(0.5 \mathrm{mg} / \mathrm{mL}\right.$ in saline) and $\mathrm{Al}(\mathrm{OH})_{3}(20 \mathrm{mg} / \mathrm{mL}$ in saline) were mixed in a 1:1 ratio, and mice were then sensitized with an intraperitoneal injection at a dosage of $0.2 \mathrm{~mL} / \mathrm{mouse}$ with the OVA mixture on days $1,7,14$ to induce systemic sensitization and the group 1 were administrated with saline. On days 22 to 32, TZD (6.4, 3.2, and $1.6 \mathrm{~g} / \mathrm{kg}), \mathrm{LTD}(2 \mathrm{mg} / \mathrm{kg})$ and a control vehicle (distilled water) was orally administrated by irrigation after 1\%OVA intranasal challenge $(20 \mu \mathrm{L}$ each nasal cavity) for 10 consecutive days. At the end of experiment, Mice were sacrificed $24 \mathrm{~h}$ after the last OVA challenge and samples were collected. The spleen weight was recorded to assess spleen/body weight ratio.

\subsection{Evaluation of Nasal Symptoms}

The number of nose rubbing movements and sneezing over 10 min duration followed by the last allergen instillation was recorded with respect to the control group.

\subsection{Histological examination}

For nasal histopathology examination, the heads were fixed in 4\% Paraformaldehyde for 3 days at $23 \pm$ $2^{\circ} \mathrm{C}$ and decalcified in an 10\% EDTA (Sigma-Aldrich; Merck KGaA) decalcifying solution for 20 days at room temperature. The samples were dehydrated with a series of ethyl alcohol and xylene, then embedded in paraffin. The sections were cut at $4 \mu \mathrm{m}$ thickness. Then, the sections were stained with hematoxylin and eosin (H\&E) (Sigma, St. Louis, MO) or toluidine blue (Sigma-Aldrich; Merck KGaA) to assess histopathological changes. Epithelial damage analysis was randomly selected using high-power fields at $400 \times$ magnification.

\subsection{Measurement of Immunoglobulins and Cytokine Levels Using ELISA}

Serum samples were collected from eyeball blood in anesthetized mice and stored at $-80^{\circ} \mathrm{C}$ until use. The levels of anti-OVA specific IgE (TSZ, Shanghai Enzyme-linked Biotechnology Co., Ltd., China) and histamine (Cloud-Clone CORP. Wuhan, China) were quantified using ELISA kits according to the manufacturers' instructions. Mouse Magnetic Luminex Assay (R\&D Systems Inc., Minneapolis, MN, USA) were used to measure cytokine levels including IL-4, IL-5, IL-13, IFN- - , IL-17 and IL-12p70 according to the manufacturer protocols.

\subsection{Protein array experiment}

Nasal tissue was collected and immediately stored at $-80^{\circ} \mathrm{C}$ after sacrifice. It was then homogenated and centrifugated to collect the supernatant for the test. To determine which related proteins are responsible for the changes in Nasal tissue, the GSM-CAA-4000 reagent kit was bought from the Ray Biotech, Inc., Guangzhou the protein array pioneer company. The operation of this experiment is carried out according to the operation of the kit.

\subsection{Bioinformatics analysis}


For individual datasets analysis, we further performed bioinformatics analysis, including Gene Ontology (GO) enrichment analysis and KEGG pathway enrichment analysis. In GO annotation and enrichment analysis, the three ontologies-biological process, molecular function, and cellular component were analyzed. The protein-protein interaction (PPI) network was composed of differentially expressed proteins and was obtained from STRING, and then constructed the PPI network by Cytoscape software and analyzed the network by MCODE app ${ }^{18}$. MCODE scores $>3$ and the default parameters were set as cutoff criteria. Finally, the CytoHubba app was used to identify PPI network hub proteins ${ }^{16}$. CytoHubba provides eleven calculation methods to identify key proteins in biological networks, and we choose the MCC method for it has a better performance ${ }^{17}$. In addition, a hierarchical clustering analysis was conducted using the R Package heatmap (https://cran.r-project.org/web/packages/cluster/). A Venn diagram was drawn online at Venny 2.1.

\subsection{Statistical analysis}

In behavioral tests, data are reported as the mean \pm standard error of the mean (SEM) as mean \pm sem. and were analyzed by one-way analysis of variance followed by Fisher's LSD post-hoc. Statistical analysis was performed with SPSS20.0 (SPSS, Armonk, New York, USA). P $<0.05$ was considered to be statistically significant.

\subsection{Molecular docking}

To verify the potential interaction, a web-based molecular docking tool, CB-Dock (http://clab.labshare.cn/cb-dock/php/) was used ${ }^{19}$. This tool was constructed based on AutoDock Vina software. Two components were selected among the core components of TZD and docked with two proteins selected from the core targets to verify the accuracy of the main components and prediction targets. The candidate composition and the target crystal structure were downloaded from the TCMSP database and RCSB protein data (https://www.wwpdb.org/) ${ }^{20}$, respectively. We uploaded these files into CB-Dock system to calculate binding score and visualize the potential poses.

\section{Results}

\subsection{Network pharmacology analysis of TZD}

\subsubsection{Screening of the active compounds and targets prediction}

A total of 280 unduplicatedly active ingredients of eleven herbal medicines in TZD were identified based on threshold values of $\mathrm{OB} \geq 30 \%$ and $\mathrm{DL} \geq 0.18$. Moreover, 240 targets of active compounds of TZD were obtained from the TCMSP database.

\subsubsection{Compound-target network construction and analysis}


In order to reflect the interactions intuitively between the active compounds of TZD and their potential targets from a systematic and holistic view, the C-T network was constructed by mapping 280 active compounds to their 240 corresponding potential targets. As shown in Fig. 1A, the network consisted of 531 nodes ( 11 herbal medicines nodes, 280 active compound nodes, and 240 compound-associated target nodes) and 4772 interaction edges. The $\mathrm{C}-\mathrm{T}$ network provided a reference to further investigate the pharmacological mechanisms of TZD.

\subsubsection{Construction of PPI network}

PPI network was established to better interpret the mechanisms of TZD in AR treatment by using STRING software. As shown in Fig. 2B, we obtained 74 overlapping targets after merging AR-related targets and active compound-related targets. A PPI network was then established by importing the overlapping targets into the STRING database. As shown in Fig. 2C, the network consisted of 72 nodes and 1610 edges. The hub targets were chosen by CytoHubba plugin with the MCC method and sequentially ordered as follows: IL6, TNF, VEGFA, IL1B, PTGS2, CCL2, CXCL8, MMP9, IL10 and IFNG as shown in Fig. 2D. We found that these hub targets are closely related to AR based on inference score.

\subsection{Effects of TZD on spleen/body weight ratio in TZD mice}

The TZD treatment increased spleen/body weight ratio significantly in AR mice. Furthermore, spleen/body weight ratio in LTD group had not significant difference compared to that of the AR group (Fig. 2A). The results demonstrated the TZD exhibited positive effects on immune organs which partially contributed to the anti-inflammatory activity ${ }^{21}$.

\subsection{Effects of TZD on nasal symptoms in AR mice}

To determine the effect of TZD on nasal symptoms, the frequencies of nasal rubbing and sneezing behaviors were evaluated for 10 min after the OVA challenge. As shown in Fig. 2B, intranasal OVA challenge to the OVA-sensitized mice induced the rubbing and sneezing responses. TZD were significantly decreased the nasal rubbing and sneezing frequencies compared to those in the AR group whereas LTD had not significant difference (Fig. 2B and 2C). These findings suggested that TZD treatment efficiently improved OVA-induced allergic nasal symptoms.

\subsection{Effects of TZD on nasal mucosa histology in AR mice}

TZD treatment improved the histological changes in the nasal tissue of mice with AR. HE-stained sections of nasal mucosa showed that the model group exhibited typical features of AR in the mucosa and submucosa, follicles were enlarged and massively infiltrated by inflammatory cell infiltration. Whereas the nasal cavity of mice in, the mice in the TZD group showed markedly reduced edema and inflammatory cell infiltration. As expected, the LTD group also displayed marked improvements in the nasal mucosa pathology compared with AR group (Fig. 3A). Toluidine blue staining was used for detection of mast cells. Compared with those in the control group, the numbers of mast cells in the nasal 
mucosa and lung tissues were significantly increased. However, the TZD group was observed a progressive decrease of mast cells in the superficial nasal epithelium (Fig. 3B).

\subsection{Effects of TZD on level of OVA-specific IgE and histamine in serum}

It is well-known that antibodies and histamine play a key role in the immune system. The anti-OVA specific IgE and histamine in serum were determined to evaluate the effect of TZD on allergic inflammatory responses. There was a significant up-regulation of anti-OVA-specific IgE (Fig. 4A) and histamine (Fig. 4B) in AR group compared with those in the naive group. In contrast, TZD and LTD groups administration resulted in lower levels of anti-OVA IgE and histamine than the levels observed in the AR group. Therefore, these results suggest that the inhibition of anti-OVA-specific IgE and histamine release is associated with the anti-allergic effect of TZD.

\subsection{TZD Modulated the Balance of Th1/Th2/Th17- Associated Cytokines in serum}

We examined the levels of Th1 (IFN-y, IL-12), Th2 (IL-4, IL-5, IL-13) and Th17 (IL-17) cytokines in serum to investigate the effect of TZD on the modulation of T helper cells responses (Fig. 5). In serum of AR group, the levels of IL-4, IL-5, IL-13 and IL-17 were significantly elevated; IL-12 and IFN-y levels were significantly reduced compared to those in the control group. In contrast, TZD oral administrations significantly downregulated IL-4 and IL-13 levels compared to the levels observed in the OVA group. In addition, TZD-treated mice had significantly up-regulated IL-12, INF- $\gamma$ in serum. We investigated whether TZD regulates Th2associated cytokine in the splenocytes via OVA-recall response. TZD enhanced cell proliferation and significantly inhibited the secretion of IL-4 and IL-13. These results indicated that TZD oral dministration could reverse an abnormal allergic immune response and regulate the balance of Th1/Th2/Th17 responses by enhancing the Th1 response and by suppressing the Th2 and Th17 responses.

\subsection{Mechanisms of TZD with proteomic Analysis}

To explore the differentially expressed proteins in the nasal mucosa, we selected potential targets with 1.2-fold change differences and P.value $<0.05$ as the screening criteria. A total of 41 proteins were found as differentially expressed proteins (DEPs) in the AR model group and TZD group.

\subsubsection{Clustering Analysis}

By plotting the cluster heatmap, the global expression of these 6 samples and 41 DEPs can be presented from a macroscopic perspective. Fig. 6A visually shows that the expression of DEPs in AR group and TZD group are significantly different.

\subsubsection{GO Enrichment Analysis}

The experiment screened a total of 1077 biological processes (BP) and 75 molecular functions (MF). BP involves the positive regulation of response to external stimuli, the positive regulation of peptidyl tyrosine 
phosphorylation, cell chemotaxis, and epithelial cells. Proliferation, see Fig. $6 \mathrm{C}$ and MF related to receptor ligand activity, cytokine activity, growth factor activity, cytokine receptor binding and growth factor receptor binding. Figure 6D shows some results of this experiment (BP in $6 \mathrm{C})$. And MF in $6 \mathrm{D}$ are sorted in descending order according to the P.adjust of $\mathrm{GO}$ analysis). The cellular components (CC) affected by TZD in Fig. 6E mainly include extracellular matrix, receptor complex, and collagen-containing extracellular matrix.

\subsubsection{KEGG Enrichment Analysis}

KEGG pathway enrichment results are presented in Fig. 6B. As shown in Fig. 6B, top 20 signaling pathways enriched by KEGG included PI3K-Akt signaling pathway, MAPK signaling pathway, Cytokinecytokine receptor interaction, EGFR tyrosine kinase inhibitor resistance, Ras signaling pathway, NF-kappa B signaling pathway, Rap1 signaling pathway, Cell adhesion molecules, Asthma and IL-17 signaling pathway.

\subsubsection{Selection of key DEPs}

As shown in Fig. 7A, there were 41 DEPs between the TZD and model groups, 71 DEPs between the control and model groups. Taking the intersection of the two, we ultimately obtained 11 proteins. From Fig. 7A, we see 6 proteins were decreased in the model group and increased in the TZD group. 5 proteins changes in opposite directions, namely increased in the model group and decreased in the TZD group.

We construct PPI network of the related proteins based on the String further (Fig. 7B). And then we analyzed the PPI network by MCODE and CytoHubba to identify the key proteins in the network. The proteins were chosen by CytoHubba plugin with the MCC method and sequentially ordered as follows: IL6 and CD 40. In addition, we verified protein expression by Protein array. As shown in Fig. 7C, compared to the control group, protein levels of IL- 6 and CD 40 were significantly up-regulated in the model group, then down-regulated in the TZD group compared to the AR group.

\subsection{Docking results analysis}

Integrate analysis of systems pharmacology and proteomics was performed, and the two components with the highest content of indigo were selected for molecular docking verification, namely quercetin and wogonin. According to TCMSP database, there is no directly related compound to CD40 but CD40 ligand (CD40L), however CD40 is closely associated with CD40L as its receptor, so we add CD40L for molecular docking verification. CD40, CD40L, and IL6 were selected from the core targets to download the crystal structures from RCSB protein data.

As shown in Fig. 8, quercetin and wogonin are more easier to combine with the key targets and play an effective role in AR. Quercetin and wogonin could bind to the hub targets stably through the analysis of hydrogen bonds and their binding sites. The logarithms of free binding energy calculated by CB-Dock of CD40-Quercetin, C CD40L-Quercetin, IL-6-Quercetin and IL6-Wogonin were -6.2, -7.6, -7.5 and -7.1 $\mathrm{kcal} / \mathrm{mol}$. The docking scores and the spatial structure suggested that multiple active compounds of TZD 
were able to bind tightly to the key targets, which further indicated that the specific therapeutic effect of TZD in the treatment of AR.

\section{Discussion}

TCM formulas always contain multiple herbs (multiple components) and exert a holistic influence through the "multi-component, multi-target, multi-effect" mechanism, which led to the difficulty of studying it. Integrating systems pharmacology and proteomics and molecular docking evaluation, we identified multiple bio-active compounds, and potential therapeutic targets of TZD against AR.

TZD is characterized by sneezing, nasal congestion, nasal itching and nasal discharge and is caused by lgE-mediated reactions to inhaled allergens. These immune reactions involve mucosal inflammation that is driven by type 2 cells ${ }^{22}$. AR seems to be the consequence of environmental exposures acting on a predisposed genetic background ${ }^{23}$. The main goals of improvement of treatment schemes in AIT are increasing safety while maintaining or even increasing efficacy and many new products are in development ${ }^{24-27}$. However, the vast majority of previous attempts failed because safety issues or lack of efficacy was observed. Future directions for conventional AIT include use of adjuvants, including vitamin D, Toll-like receptor ligand agonists, biologics ${ }^{28}$ or probiotics ${ }^{29}$. There are several attempts like molecular allergy vaccines acting on B cells or T cells but none has produced convincing results ${ }^{30}$. However, we require more effective therapy to treat AR with few side effects. In this study, we verified that TZD could be used to treat AR. Our present study clearly demonstrates that TZD suppresses the progression of TZD induced by OVA. This suggests that TZD might be a useful drug for the treatment of AR.

In our study, we found an elevation of serum OVA-specific IgE level and histamine ${ }^{31}$ release in OVA group, but not in TZD-treated mice. Furthermore, the down-regulation of serum OVA-specific antibodies and histamine levels were consistent with the reduction of allergic symptoms including sneezing and rubbing ${ }^{32}$ scores in TZD groups. These results indicated that TZD oral administrations could suppress the allergic responses in the early phase of AR. At the late-phase reaction of AR, eosinophils release eosinophil cationic protein, eotaxin, and proinflammatory mediators that cause mucosal damage and edema $^{33}$. OVA-exposed mice showed an increased accumulation of eosinophils in nasal mucosa layers, according to results, the nasal mucosa layers were swelling with high infiltration of inflammatory cells. Meanwhile, TZD-treated mice were effective in suppressing nasal eosinophilia and nasal thickness. Also, the decrease of nasal eosinophils was correlated with the serum histamine reduction which took place in the early-phase after treatment. According to these results, suggesting that TZD oral treatments could inhibit the allergic responses in both early-phase and late-phase of AR. TZD remarkably inhibited rhinitis symptoms including the clinical scores, the morphological changes of nasal mucosa, and the histamine release in OVA-sensitized mice. 
AR is further progressed by Th2 and Th17 cytokines such as IL-4, IL-13 and IL-17 ${ }^{34}$. These cytokines maintain and aggravate an inflammatory condition in the nasal mucosa by inducing the infiltration of inflammatory cells and the disruption of an intact mucosal barrier ${ }^{35}$. Antagonizing IL-4 and IL-13 invitro and anti-IL-4 treatment in mice prevented epithelial barrier disruption and hence represents a possible strategic target for breaking persistent nasal inflammation in $\mathrm{AR}^{36}$.

The PI3K/Akt signaling pathway is important for cell growth, differentiation, metabolism, survival and apoptosis ${ }^{37}$. Some previous studies have demonstrated that the PI3K/Akt signaling pathway participated in the regulation on Mast Cells degranulation ${ }^{38,39}$, which serve a crucial role in allergic diseases, including bronchial asthma ${ }^{40}$, allergic rhinitis ${ }^{41}$ and hypersensitivity ${ }^{42}$. NF-KB and MAPK pathways are also two important inflammatory related signaling pathways in mast cells ${ }^{43,44}$. Our results showed TZD can exert anti-allergic effect through inhibiting allergic and inflammatory related pathways.

Studies show that the increase in IL-6 indicates the presence of inflammation. IL- 6 can promote Th2 cell phenotypes and increases congestion of nose ${ }^{45}$. Moreover, elevated levels of IL- 6 in allergic rhinitis patients can result in fatigue and other nonspecific mental symptoms, such as stress and depression ${ }^{46}$. CD40 is an important costimulatory molecule in immunity, which plays a role in tissue remodelling in $A R^{47}$. It is expressed on B cells, dendritic cells (DCs), macrophages, monocytes, epithelial cells, endothelial cells, mesenchymal cells, and platelets. The CD40 pathway is an important costimulatory pathway in immunity. The interaction of CD40 and its ligand is required for effective adaptive and innate immune responses as it induces several immunostimulatory events, including the licensing of antigenpresenting cells (APCs), T cell activation, and B cell class switching ${ }^{48}$. CD40 siRNA therapy shows promise for chronic TZD as it significantly attenuated allergic symptoms and Th2-related inflammation ${ }^{47}$, which is in agreement with our research. These results suggested IL- 6 and CD 40 maybe potential therapeutic targets of TZD.

Quercetin is a naturally occurring polyphenol flavonoid which is rich in antioxidants. It has anti-allergic functions that are known for inhibiting histamine production and pro-inflammatory mediators. Quercetin can regulate the $\mathrm{Th} 1 / \mathrm{Th} 2$ stability ${ }^{49}$, and decrease the antigen-specific $\operatorname{lgE}$ antibody releasing by $\mathrm{B}$ cells ${ }^{50}$. Studies showed that quercetin could inhibits IgE-mediated like allergic conjunctivitis ${ }^{51}$ and atopic dermatitis ${ }^{52}$. Wogonin is a group of polyphenols ubiquitous in plants. It has been shown to have very potent antioxidative effect on the peroxidation of phospholipids bilayers from free radical attack, inhibitory effect on the actions of IgE production, and enhancing differentiation of Th0 cells to become Th1 cells in rats. Wogonin induces $\mathrm{HO} 1$ expression ${ }^{53}$ and eosinophil apoptosi ${ }^{54}$ s, suggesting that it has therapeutic potential for the treatment of allergic inflammation in humans. Quercetin and Wogonin exhibited superior binding affinity in molecular docking, indicating that they might play a crucial role in the anti-AR effects of TZD, which may be the potentially quality control components.

\section{Conclusions}


Collectively, the results of systems pharmacology and proteomics indicated that TZD can effectively exert anti-AR activities through synergistic effects of multiple compounds, targets, and signaling pathways.

Our study of the AR mouse model demonstrated that TZD exerts a potent anti-inflammatory effect, which ameliorated the rhinitis symptoms, reduced the nasal histopathology, inhibited the release of proinflammatory mediators, and regulated the differentiation of Th1 and Th2 cells. These findings provide support for the use of TZD in the treatment of AR and may provide a promising strategy for immunotherapy in allergic rhinitis. The results of proteomics indicated that TZD may exert anti-AR activities through synergistic effects of targets, and signaling pathways. Taken together, these findings uncover the underlying mechanism of TZD against AR and provide a scientific method for the rational development of traditional Chinese medicine.

\section{Abbreviations}

TZD

Tuomin-Zhiti-Decoction

AR

Allergic rhinitis

OVA

ovalbumin

$\lg \mathrm{E}$

immunoglobulin $\mathrm{E}$

DEPs

differentially expressed proteins

AIT

Allergen immunotherapy

IL-4

Interleukin-4

IL-5

Interleukin-5

IL-13

Interleukin-13

IFN-Y

Interferon gamma

IL-17

Interleukin-17

IL-12p70

Interleukin-12 p70

IL-6

Interleukin-6

IL-10 
Interleukin-10

CD40

Tumor necrosis factor receptor superfamily member 5

CD40L

CD40 ligand

IL-1B

Interleukin-1B

TNF

Tumor necrosis factor

PI3K

Phosphoinositide 3-kinase

AKT

Serine/threonine-protein kinase

CCL2

$\mathrm{C}-\mathrm{C}$ motif chemokine 2

VEGFA

Vascular endothelial growth factor $A$

NF-KB

Nuclear factor kappa-B

PTGS2

Prostaglandin $\mathrm{G} / \mathrm{H}$ synthase 2

CXCL8

C-X-C motif chemokine 8

MAPK

Mitogen-activated protein kinase

MMP9

Matrix metalloproteinase- 9

\section{Declarations}

\section{Acknowledgements}

Not applicable.

\section{Authors' contributions}

JW and QW designed the study; JC and MZ performed the experiment; JC analyzed the data and wrote the article; $M Z$ revised the manuscript; $Y Z$ participated part of the experiments. All authors read and approved the final manuscript.

\section{Funding}


This work was supported by China Postdoctoral Science Foundation (2020M670235) and Innovation Team and Talents Cultivation Program of National Administration of Traditional Chinese Medicine. (No: ZYYCXTD-C-202001)

\section{Availability of data and materials}

The datasets used and/or analyzed during the current study are available from the corresponding author upon reasonable request.

\section{Ethics approval and consent to participate}

The animal care and experimental procedures used in this study were approved by the Institutional Animal Care and Use Committee of Beijing University of Chinese Medicine.

\section{Consent for publication}

Not applicable.

\section{Competing interests}

These authors declare that there are no conflicts of interest regarding this work.

\section{References}

1. Brozek JL, Bousquet J, Baena-Cagnani CE, Bonini S, Canonica GW, Casale TB, et al. Allergic Rhinitis and its Impact on Asthma (ARIA) guidelines: 2010 revision. J Allergy Clin Immunol 2010; 126 (3): 466-476. doi: 10.1016/j.jaci.2010.06.047.

2. Wise SK, Lin SY, Toskala E, Orlandi RR, Akdis CA, Alt JA, et al. International Consensus Statement on Allergy and Rhinology: Allergic Rhinitis. Int Forum Allergy Rhinol 2018; 8 (2): 108-352. doi: 10.1002/alr.22073.

3. Brożek JL, Bousquet J, Agache I, Agarwal A, Bachert C, Bosnic-Anticevich S, et al. Allergic Rhinitis and its Impact on Asthma (ARIA) guidelines-2016 revision. J Allergy Clin Immunol 2017; 140 (4): 950-958. doi: 10.1016/j.jaci.2017.03.050.

4. Zuberbier T, Lötvall J, Simoens S, Subramanian SV, Church MK. Economic burden of inadequate management of allergic diseases in the European Union: a GA(2) LEN review. Allergy 2014; 69 (10): 1275-1279. doi: 10.1111/all.12470.

5. Bui TT, Piao CH, Hyeon E, Fan Y, Choi DW, Jung SY, et al. Preventive Effect of Bupleurum chinense on Nasal Inflammation via Suppressing T Helper Type 2, Eosinophil and Mast Cell Activation. Am J Chin Med 2019; 47 (2): 405-421. doi: 10.1142/s0192415x19500204.

6. Kim HY, Kim J, Jeong HJ, Kim HM. Potential anti-inflammatory effect of Madi-Ryuk and its active ingredient tannic acid on allergic rhinitis. Mol Immunol 2019; 114 362-368. doi:

10.1016/j.molimm.2019.08.013. 
7. Lin $B$, Cai $B$, Wang H. Honeysuckle extract relieves ovalbumin-induced allergic rhinitis by inhibiting AR-induced inflammation and autoimmunity. Biosci Rep 2019; 39 (7): doi: 10.1042/bsr20190673.

8. Ru J, Li P, Wang J, Zhou W, Li B, Huang C, et al. TCMSP: a database of systems pharmacology for drug discovery from herbal medicines. J Cheminform 2014; 6 13. doi: 10.1186/1758-2946-6-13.

9. Fang S, Dong L, Liu L, Guo J, Zhao L, Zhang J, et al. HERB: a high-throughput experiment- and reference-guided database of traditional Chinese medicine. Nucleic Acids Res 2021; 49 (D1): D1197d1206. doi: 10.1093/nar/gkaa1063.

10. UniProt: the universal protein knowledgebase in 2021. Nucleic Acids Res 2021; 49 (D1): D480-d489. doi: 10.1093/nar/gkaa1100.

11. Amberger JS, Bocchini CA, Schiettecatte F, Scott AF, Hamosh A. OMIM.org: Online Mendelian Inheritance in Man (OMIM $\left.{ }^{\circledR}\right)$, an online catalog of human genes and genetic disorders. Nucleic Acids Res 2015; 43 (Database issue): D789-798. doi: 10.1093/nar/gku1205.

12. Piñero J, Saüch J, Sanz F, Furlong LI. The DisGeNET cytoscape app: Exploring and visualizing disease genomics data. Comput Struct Biotechnol J 2021; 19 2960-2967. doi: 10.1016/j.csbj.2021.05.015.

13. Safran M, Dalah I, Alexander J, Rosen N, Iny Stein T, Shmoish M, et al. GeneCards Version 3: the human gene integrator. Database (Oxford) 2010; 2010 baq020. doi: 10.1093/database/baq020.

14. Wishart DS, Feunang YD, Guo AC, Lo EJ, Marcu A, Grant JR, et al. DrugBank 5.0: a major update to the DrugBank database for 2018. Nucleic Acids Res 2018; 46 (D1): D1074-d1082. doi: 10.1093/nar/gkx1037.

15. Szklarczyk D, Gable AL, Nastou KC, Lyon D, Kirsch R, Pyysalo S, et al. The STRING database in 2021: customizable protein-protein networks, and functional characterization of user-uploaded gene/measurement sets. Nucleic Acids Res 2021; 49 (D1): D605-d612. doi: 10.1093/nar/gkaa1074.

16. Chin $\mathrm{CH}$, Chen $\mathrm{SH}, \mathrm{Wu} \mathrm{HH}, \mathrm{Ho} \mathrm{CW}$, Ko MT, Lin CY. cytoHubba: identifying hub objects and subnetworks from complex interactome. BMC Syst Biol 2014; 8 Suppl 4 (Suppl 4): S11. doi: 10.1186/1752-0509-8-s4-s11.

17. Guo C, Li Z. Bioinformatics Analysis of Key Genes and Pathways Associated with Thrombosis in Essential Thrombocythemia. Med Sci Monit 2019; 25 9262-9271. doi: 10.12659/msm.918719.

18. Pruitt KD, Maglott DR. RefSeq and LocusLink: NCBI gene-centered resources. Nucleic Acids Res 2001; 29 (1): 137-140. doi: 10.1093/nar/29.1.137.

19. Liu Y, Grimm M, Dai WT, Hou MC, Xiao ZX, Cao Y. CB-Dock: a web server for cavity detection-guided protein-ligand blind docking. Acta Pharmacol Sin 2020; 41 (1): 138-144. doi: 10.1038/s41401-0190228-6.

20. Protein Data Bank: the single global archive for 3D macromolecular structure data. Nucleic Acids Res 2019; 47 (D1): D520-d528. doi: 10.1093/nar/gky949.

21. Li W, Hu X, Wang S, Jiao Z, Sun T, Liu T, et al. Characterization and anti-tumor bioactivity of astragalus polysaccharides by immunomodulation. Int J Biol Macromol 2020; 145 985-997. doi: 10.1016/j.ijbiomac.2019.09.189. 
22. Greiner AN, Hellings PW, Rotiroti G, Scadding GK. Allergic rhinitis. Lancet 2011; 378 (9809): 21122122. doi: 10.1016/s0140-6736(11)60130-x.

23. Bousquet J, Anto JM, Bachert C, Baiardini I, Bosnic-Anticevich S, Walter Canonica G, et al. Allergic rhinitis. Nat Rev Dis Primers 2020; 6 (1): 95. doi: 10.1038/s41572-020-00227-0.

24. Dorofeeva Y, Shilovskiy I, Tulaeva I, Focke-Tejkl M, Flicker S, Kudlay D, et al. Past, present, and future of allergen immunotherapy vaccines. Allergy 2021; 76 (1): 131-149. doi: 10.1111/all.14300.

25. Pechsrichuang $P$, Jacquet $A$. Molecular approaches to allergen-specific immunotherapy: Are we so far from clinical implementation? Clin Exp Allergy 2020; 50 (5): 543-557. doi: 10.1111/cea.13588.

26. Rodríguez-Domínguez A, Berings $M$, Rohrbach A, Huang HJ, Curin M, Gevaert $P$, et al. Molecular profiling of allergen-specific antibody responses may enhance success of specific immunotherapy. $J$ Allergy Clin Immunol 2020; 146 (5): 1097-1108. doi: 10.1016/j.jaci.2020.03.029.

27. Komlósi Zl, Kovács N, Sokolowska M, van de Veen W, Akdis M, Akdis CA. Highlights of Novel Vaccination Strategies in Allergen Immunotherapy. Immunol Allergy Clin North Am 2020; 40 (1): 1524. doi: 10.1016/j.iac.2019.09.010.

28. Massanari M, Nelson H, Casale T, Busse W, Kianifard F, Geba GP, et al. Effect of pretreatment with omalizumab on the tolerability of specific immunotherapy in allergic asthma. J Allergy Clin Immunol 2010; 125 (2): 383-389. doi: 10.1016/j.jaci.2009.11.022.

29. Nelson HS. Allergy immunotherapy: Future directions for the 2020s. Allergy Asthma Proc 2020; 41 (5): 314-325. doi: 10.2500/aap.2020.41.200041.

30. Tulaeva I, Kratzer B, Campana R, Curin M, van Hage M, Karsonova A, et al. Preventive AllergenSpecific Vaccination Against Allergy: Mission Possible? Front Immunol 2020; 11 1368. doi: 10.3389/fimmu.2020.01368.

31. Galli SJ, Tsai M. IgE and mast cells in allergic disease. Nat Med 2012; 18 (5): 693-704. doi: 10.1038/nm.2755.

32. Pipet A, Botturi K, Pinot D, Vervloet D, Magnan A. Allergen-specific immunotherapy in allergic rhinitis and asthma. Mechanisms and proof of efficacy. Respir Med 2009; 103 (6): 800-812. doi: 10.1016/j.rmed.2009.01.008.

33. Humbles AA, Lloyd CM, McMillan SJ, Friend DS, Xanthou G, McKenna EE, et al. A critical role for eosinophils in allergic airways remodeling. Science 2004; 305 (5691): 1776-1779. doi: 10.1126/science. 1100283 .

34. Gon Y, Hashimoto S. Role of airway epithelial barrier dysfunction in pathogenesis of asthma. Allergol Int 2018; 67 (1): 12-17. doi: 10.1016/j.alit.2017.08.011.

35. Steelant B, Farré R, Wawrzyniak P, Belmans J, Dekimpe E, Vanheel H, et al. Impaired barrier function in patients with house dust mite-induced allergic rhinitis is accompanied by decreased occludin and zonula occludens-1 expression. J Allergy Clin Immunol 2016; 137 (4): 1043-1053.e1045. doi: 10.1016/j.jaci.2015.10.050.

36. Steelant B, Seys SF, Van Gerven L, Van Woensel M, Farré R, Wawrzyniak P, et al. Histamine and T helper cytokine-driven epithelial barrier dysfunction in allergic rhinitis. J Allergy Clin Immunol 2018; 
141 (3): 951-963.e958. doi: 10.1016/j.jaci.2017.08.039.

37. Yu JS, Cui W. Proliferation, survival and metabolism: the role of PI3K/AKT/mTOR signalling in pluripotency and cell fate determination. Development 2016; 143 (17): 3050-3060. doi: 10.1242/dev.137075.

38. Biethahn K, Orinska Z, Vigorito E, Goyeneche-Patino DA, Mirghomizadeh F, Föger N, et al. miRNA-155 controls mast cell activation by regulating the PI3KY pathway and anaphylaxis in a mouse model. Allergy 2014; 69 (6): 752-762. doi: 10.1111/all.12407.

39. Lin H, Zheng C, Li J, Yang C, Hu L. Lentiviral shRNA against KCa3.1 inhibits allergic response in allergic rhinitis and suppresses mast cell activity via PI3K/AKT signaling pathway. Sci Rep 2015; 5 13127. doi: 10.1038/srep13127.

40. Andersson C, Tufvesson E, Diamant Z, Bjermer L. Revisiting the role of the mast cell in asthma. Curr Opin Pulm Med 2016; 22 (1): 10-17. doi: 10.1097/mcp.0000000000000228.

41. Fu S, Ni S, Wang D, Hong T. Coptisine Suppresses Mast Cell Degranulation and Ovalbumin-Induced Allergic Rhinitis. Molecules 2018; 23 (11): doi: 10.3390/molecules23113039.

42. Gaudenzio N, Marichal T, Galli SJ, Reber LL. Genetic and Imaging Approaches Reveal ProInflammatory and Immunoregulatory Roles of Mast Cells in Contact Hypersensitivity. Front Immunol 2018; 9 1275. doi: 10.3389/fimmu.2018.01275.

43. Jin C, Ye K, Luan H, Liu L, Zhang R, Yang S, et al. Tussilagone inhibits allergic responses in OVAinduced allergic rhinitis guinea pigs and IgE-stimulated RBL-2H3 cells. Fitoterapia 2020; 144104496. doi: 10.1016/j.fitote.2020.104496.

44. Paiva Ferreira LKD, Paiva Ferreira LAM, Bezerra Barros GC, Mozzini Monteiro T, de Araújo Silva LA, Pereira RA, et al. MHTP, a synthetic alkaloid, attenuates combined allergic rhinitis and asthma syndrome through downregulation of the p38/ERK1/2 MAPK signaling pathway in mice. Int Immunopharmacol 2021; 96 107590. doi: 10.1016/j.intimp.2021.107590.

45. Zheng M, Wang X, Zhang L. Association between allergic and nonallergic rhinitis and obstructive sleep apnea. Curr Opin Allergy Clin Immunol 2018; 18 (1): 16-25. doi:

10.1097/aci.0000000000000414.

46. Vgontzas AN, Zoumakis E, Bixler EO, Lin HM, Follett H, Kales A, et al. Adverse effects of modest sleep restriction on sleepiness, performance, and inflammatory cytokines. J Clin Endocrinol Metab 2004; 89 (5): 2119-2126. doi: 10.1210/jc.2003-031562.

47. Cheng KJ, Zhou ML, Liu YC, Wang C, Xu YY. The Role of CD40 in Allergic Rhinitis and Airway Remodelling. Mediators Inflamm 2021; 2021 6694109. doi: 10.1155/2021/6694109.

48. Pinelli DF, Ford ML. Novel insights into anti-CD40/CD154 immunotherapy in transplant tolerance. Immunotherapy 2015; 7 (4): 399-410. doi: 10.2217/imt.15.1.

49. Zhang T, Hu Z, Cheng Y, Xu H, Velickovic TC, He K, et al. Changes in Allergenicity of Ovalbumin in Vitro and in Vivo on Conjugation with Quercetin. J Agric Food Chem 2020; 68 (13): 4027-4035. doi: 10.1021/acs.jafc.0c00461. 
50. Jafarinia M, Sadat Hosseini M, Kasiri N, Fazel N, Fathi F, Ganjalikhani Hakemi M, et al. Quercetin with the potential effect on allergic diseases. Allergy Asthma Clin Immunol 2020; 16 36. doi: 10.1186/s13223-020-00434-0.

51. Ding Y, Li C, Zhang Y, Ma P, Zhao T, Che D, et al. Quercetin as a Lyn kinase inhibitor inhibits lgEmediated allergic conjunctivitis. Food Chem Toxicol 2020; 135 110924. doi: 10.1016/j.fct.2019.110924.

52. Hou DD, Zhang W, Gao YL, Sun YZ, Wang HX, Qi RQ, et al. Anti-inflammatory effects of quercetin in a mouse model of MC903-induced atopic dermatitis. Int Immunopharmacol 2019; 74 105676. doi: 10.1016/j.intimp.2019.105676.

53. Lee BS, Shim SM, Heo J, Pae HO, Seo BY, Han SY, et al. Wogonin suppresses TARC expression induced by mite antigen via heme oxygenase 1 in human keratinocytes. Suppressive effect of wogonin on mite antigen-induced TARC expression. J Dermatol Sci 2007; 46 (1): 31-40. doi: 10.1016/j.jdermsci.2007.01.001.

54. Lucas CD, Dorward DA, Sharma S, Rennie J, Felton JM, Alessandri AL, et al. Wogonin induces eosinophil apoptosis and attenuates allergic airway inflammation. Am J Respir Crit Care Med 2015; 191 (6): 626-636. doi: 10.1164/rccm.201408-15650C.

\section{Figures}


A

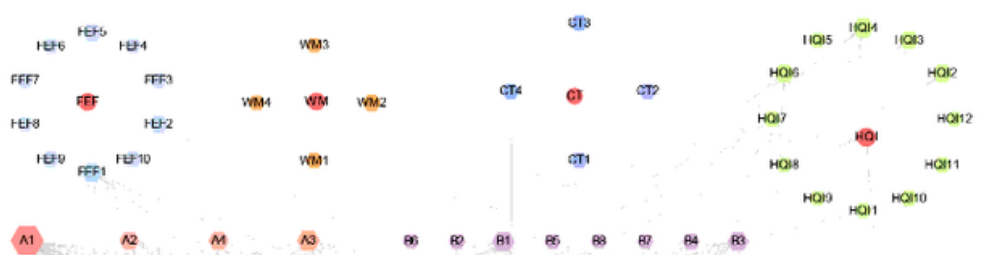

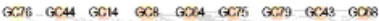

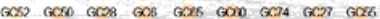

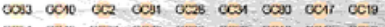

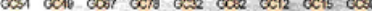

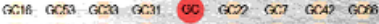

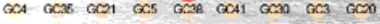

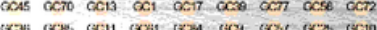

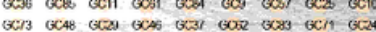

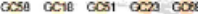

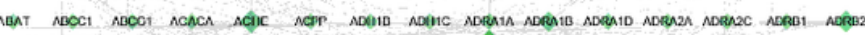

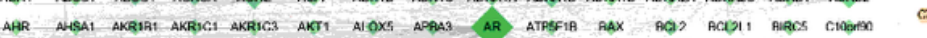

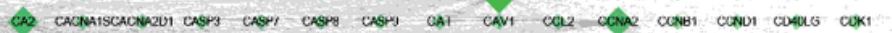

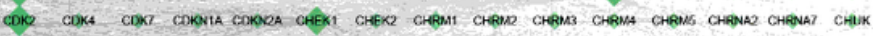

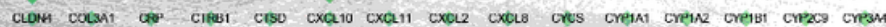

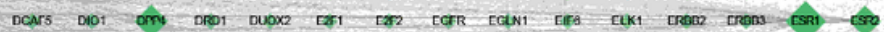

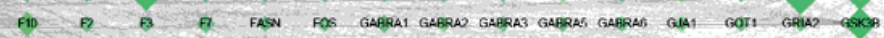

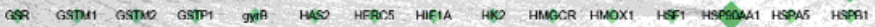

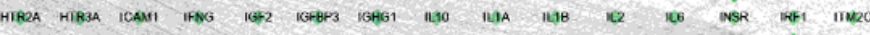

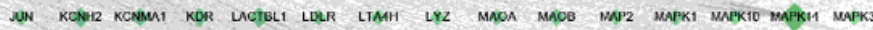
$1259 \quad \begin{array}{llllll}175 & 1743 \quad 1730 & 1741 \quad 1727 \quad 181\end{array}$

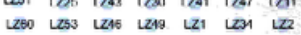

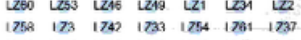

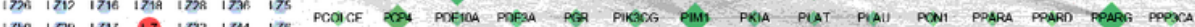

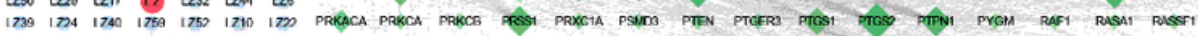

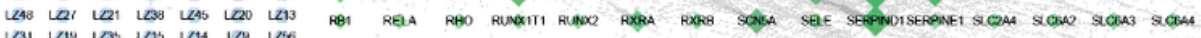
$2757 \quad 177 \quad 179 \quad 1780 \quad 174 \quad 1703 \quad 105$

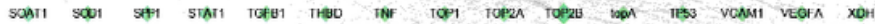
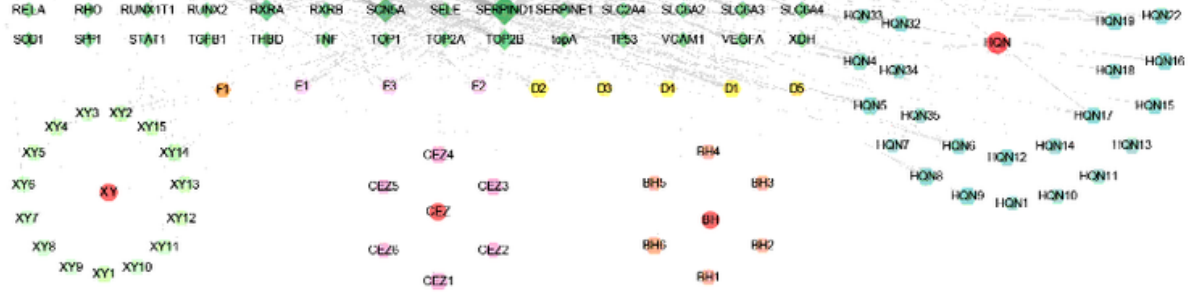

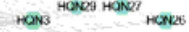

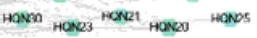

ONBIIIONOSO
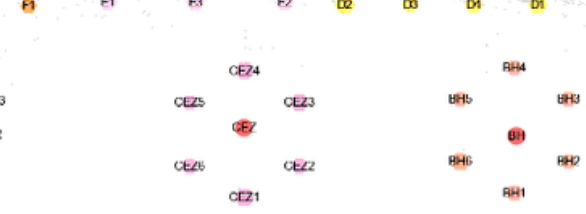

\section{B}

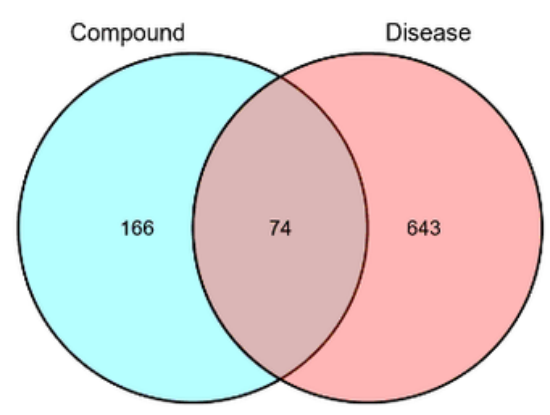

C

D
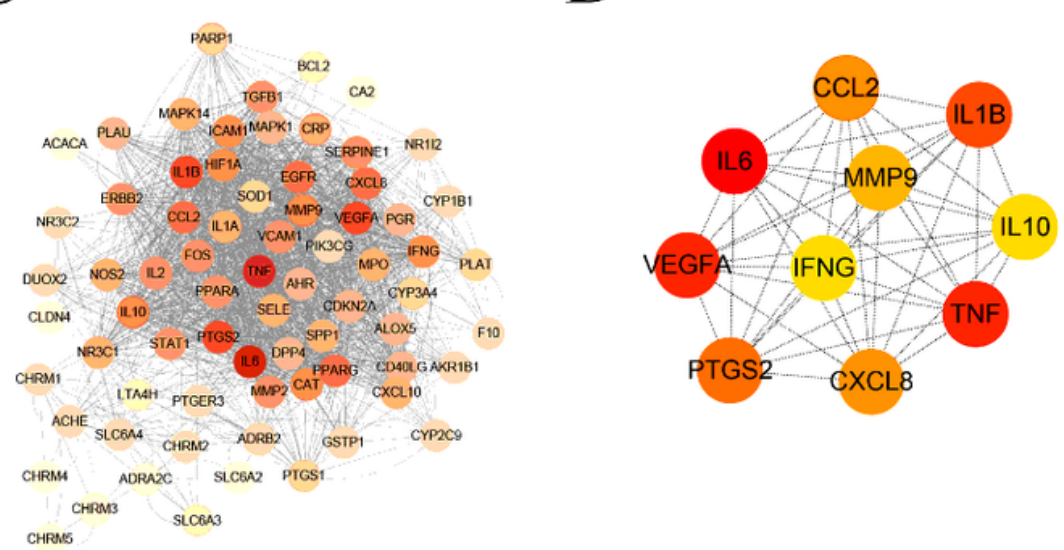

Figure 1

TZD potential target-AR target network and analysis. A: Compound-target (C-T) network of TZD. B: The Venn diagram shows 74 overlapping targets between AR-related targets and active compound-related targets. C: Protein-protein interaction (PPI) network of the 74 overlapping targets. D: The PPI of hub overlapping target. 
A

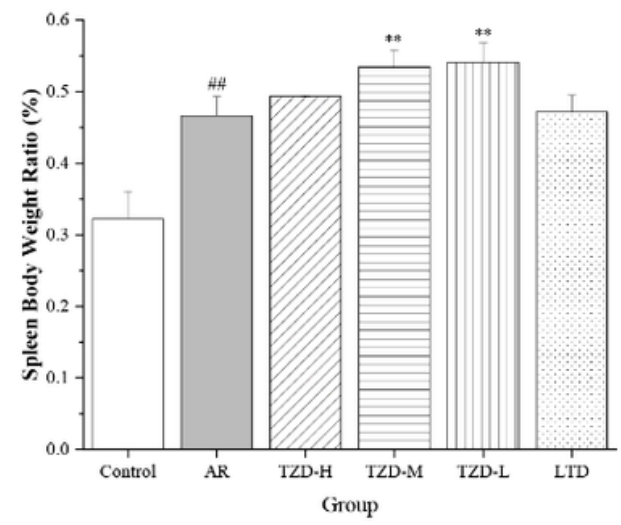

B

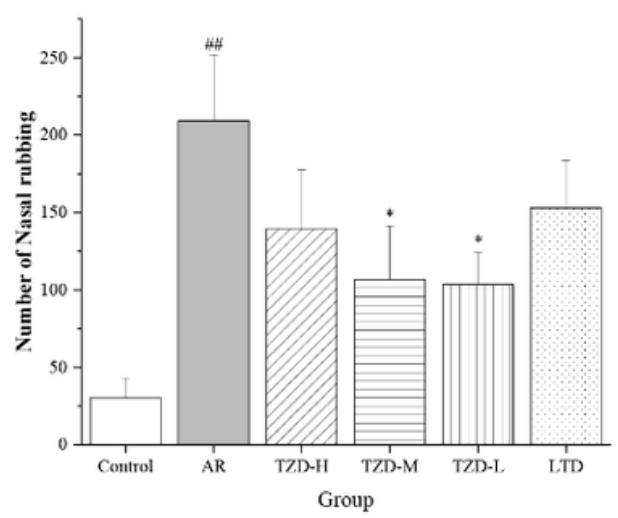

$\mathrm{C}$

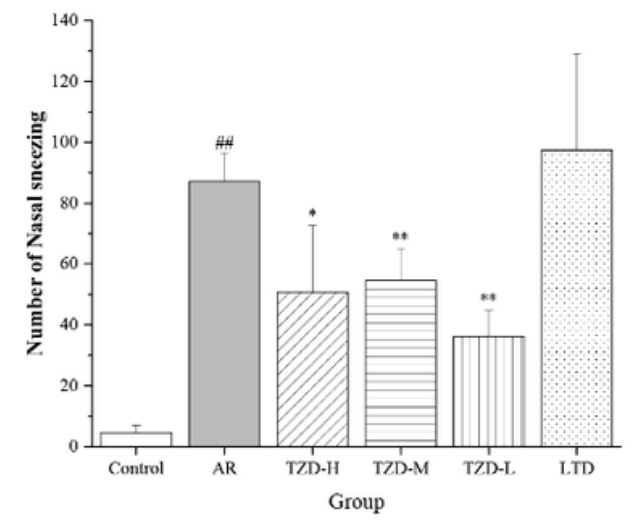

\section{Figure 2}

Effect of TZD on spleen index (A) and effect of TZD on the nasal symptoms in OVA-induced AR mouse model. The frequencies of total rubbing $(B)$ and sneezing $(C)$ numbers by counting that occurred in the 10 min after the OVA challenge. $\# P<0.05, \# \# P<0.01$, compared with the control group. ${ }^{*} P<0.05,{ }^{*} P<0.01$, compared with AR group.

展

\section{Figure 3}

Effects of TZD on histological changes of nasal mucosa in OVA- sensitized mice. (A) The histological changes of nasal mucosa were observed by microscope after H\&E staining (400X). (B) Infiltration of mast cells in nasal mucosa after Toluidine Blue staining (400X).

A

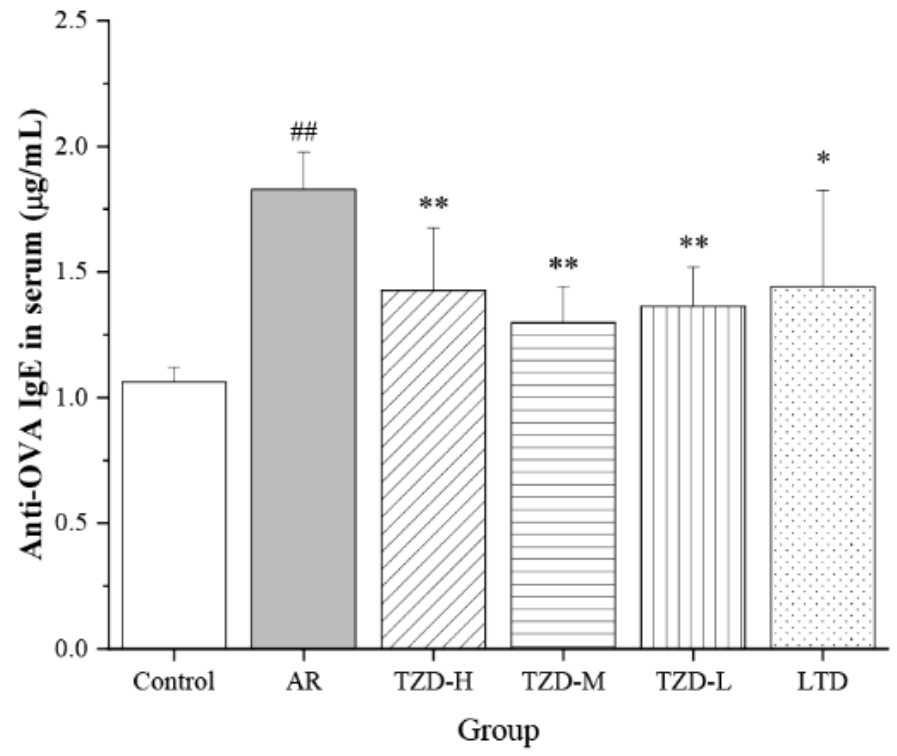

B

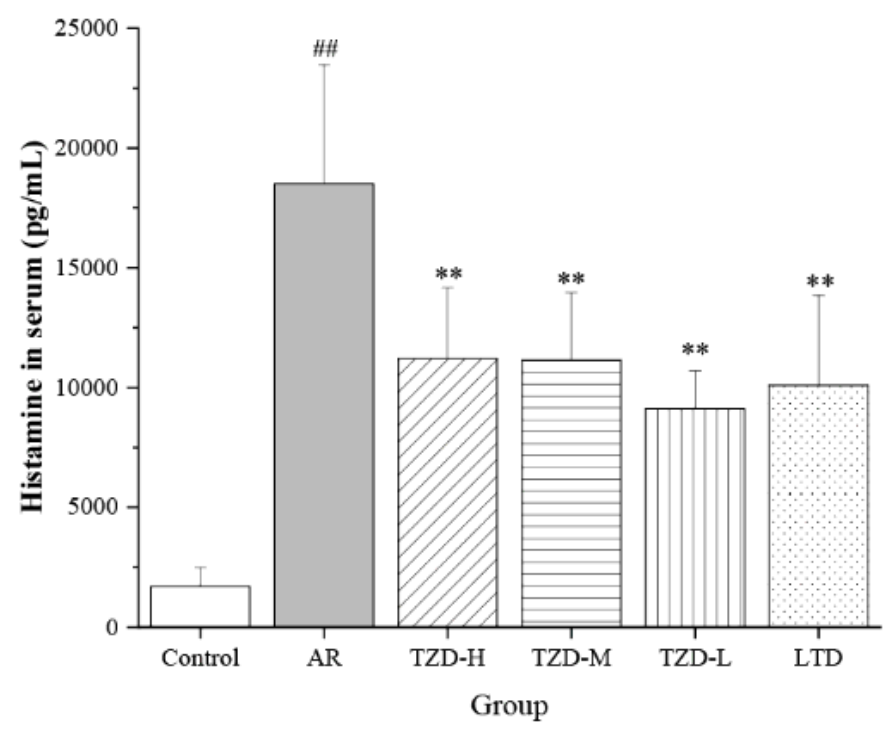

Figure 4 
TZD regulated the levels of serum anti-OVA specific antibodies and reduced mast cell histamine release. TZD-treated mice down-regulated the levels of anti-OVA specific $\lg E(A)$. Histamine level was examined in serum (B). \#P<0.05, \#\#P<0.01, compared with the control group. ${ }^{*} P<0.05, * * P<0.01$, compared with $A R$ group.

A

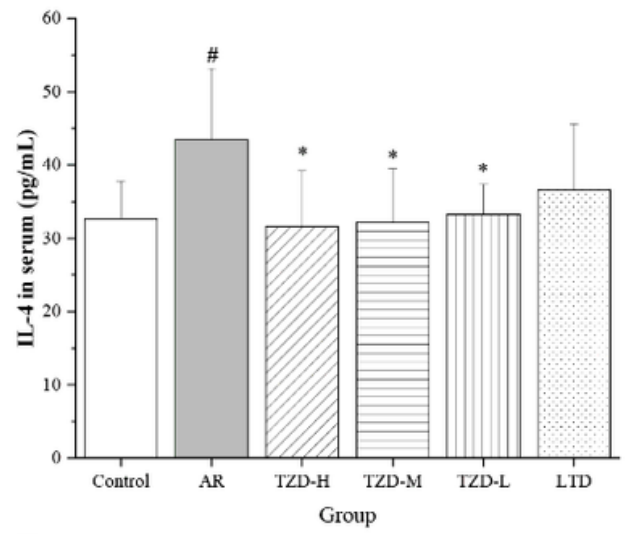

$\mathrm{D}$

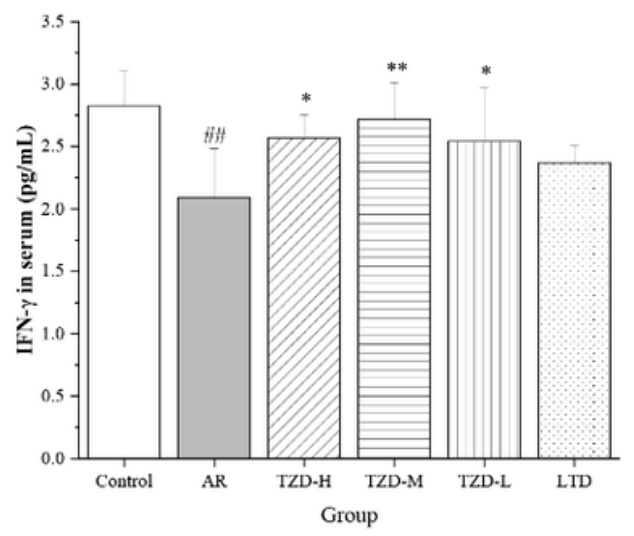

B

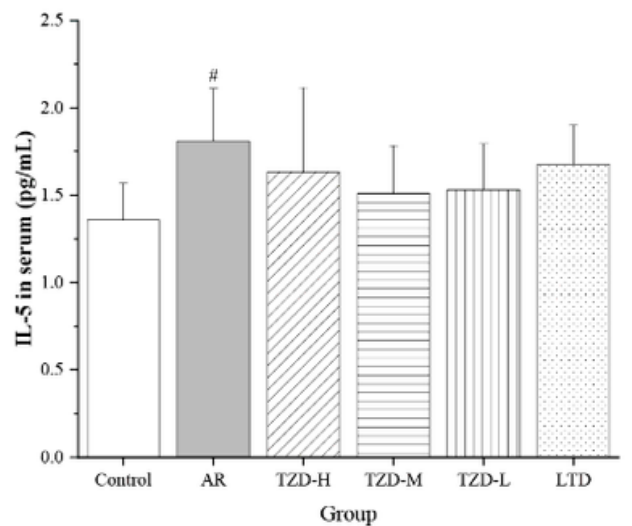

$\mathrm{E}$

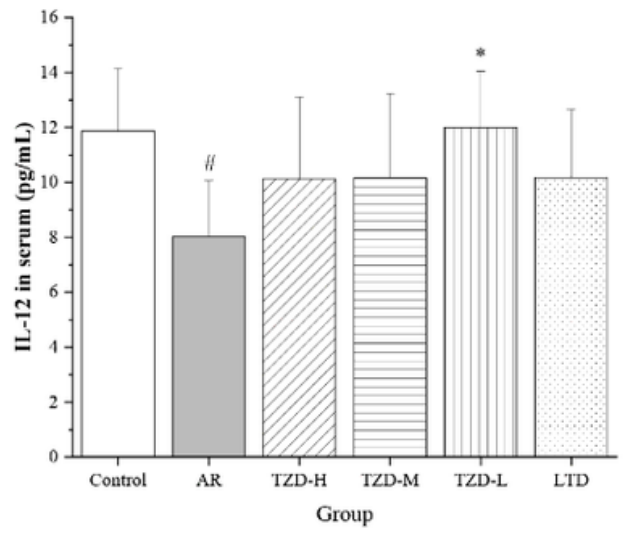

$\mathrm{C}$

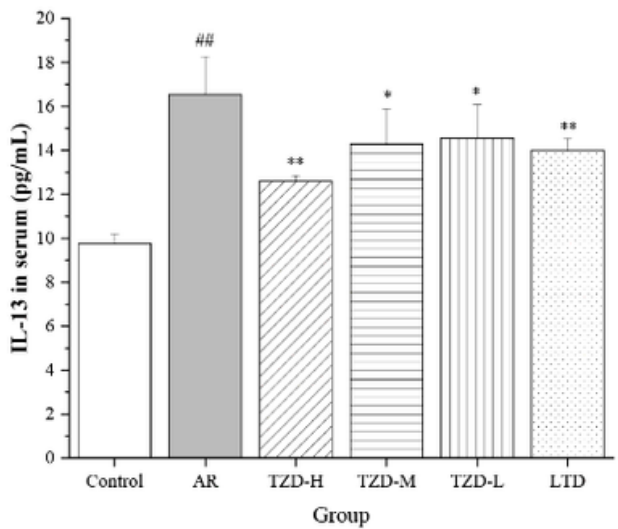

$\mathrm{F}$

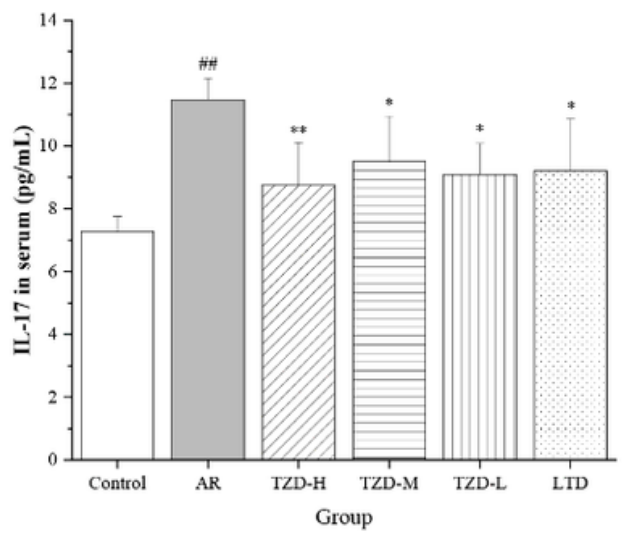

Figure 5

TZD modulated the balance of Th1/Th2/IL17-associated cytokines in serum. The Th2-related cytokines were down-regulated, while the Th1 and Th17-related cytokines were up-regulated in serum. The values represent the mean $\pm S E$ ( $n=6 /$ group). Significant differences at \#P<0.05, \#\#P<0.01 compared with the Control group. ${ }^{*} \mathrm{P}<0.05,{ }^{\star} * \mathrm{P}<0.01$, compared with the AR group. 
A
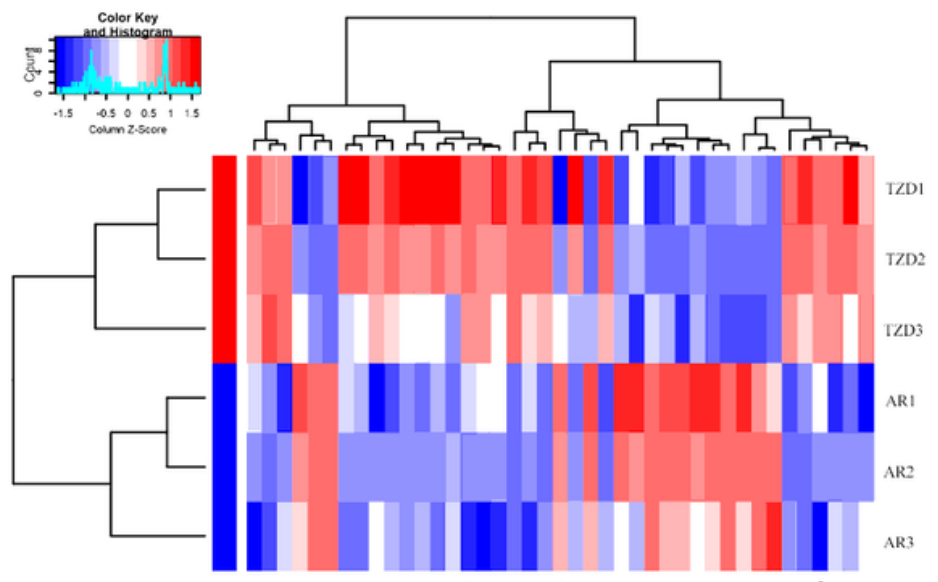

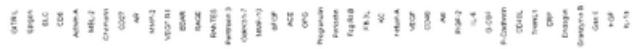

$\mathrm{C}$

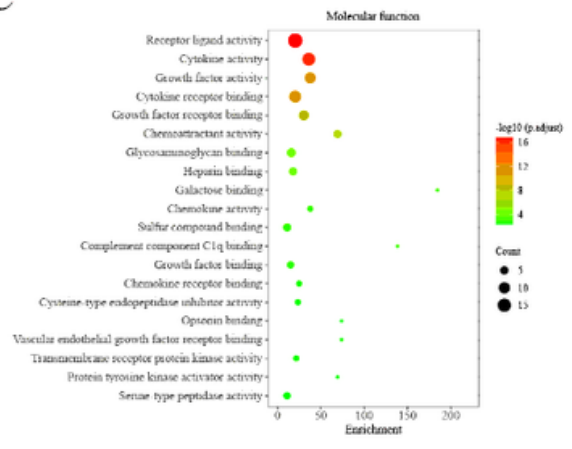

$\mathrm{D}$
$\mathrm{B}$
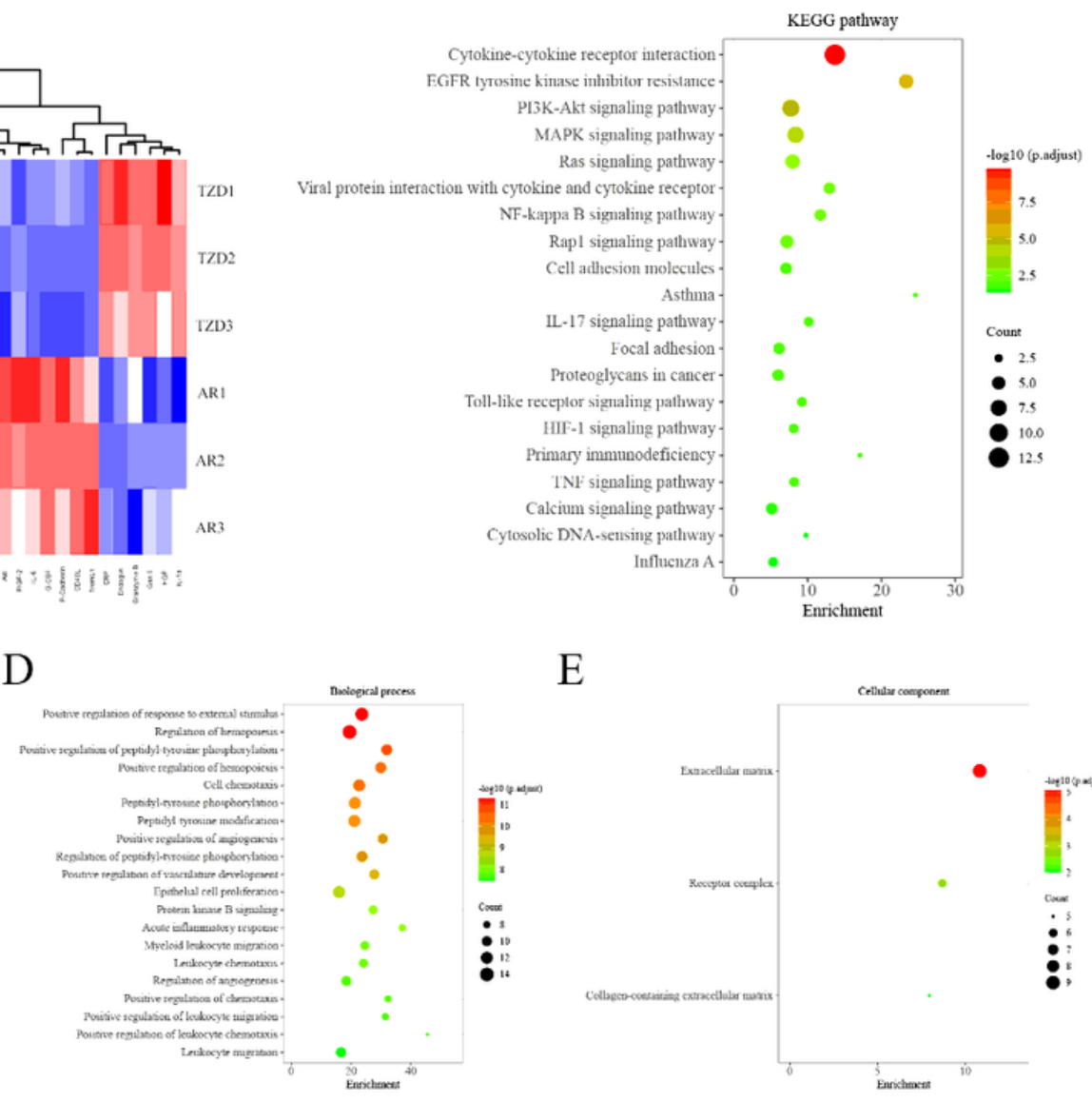

$\mathrm{E}$

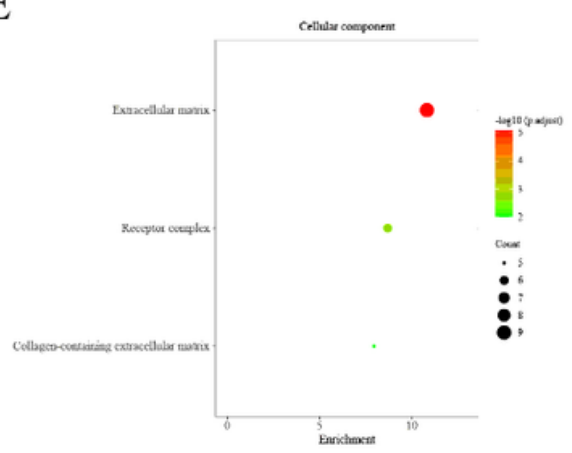

Figure 6

Clustering analysis of the differentially expressed proteins between TZD group and AR group(A). KEGG and GO analysis of differentially expressed proteins after treatment with TZD. (B) KEGG pathway. (C) Molecular function. (D) Biological process. (E) Cellular component. 
A

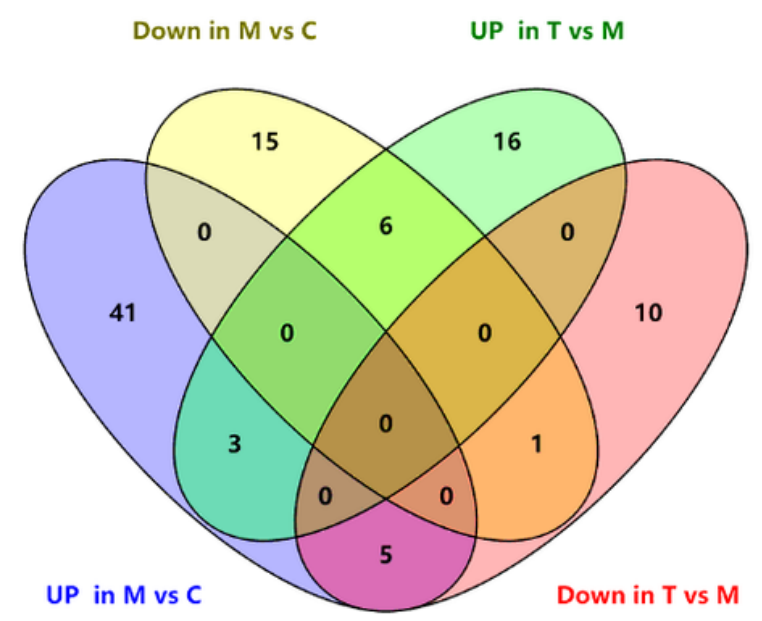

C

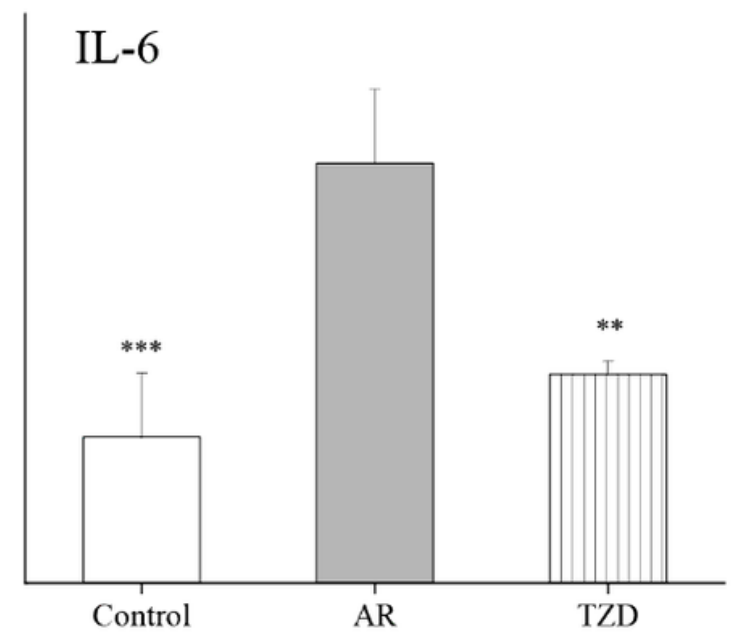

B

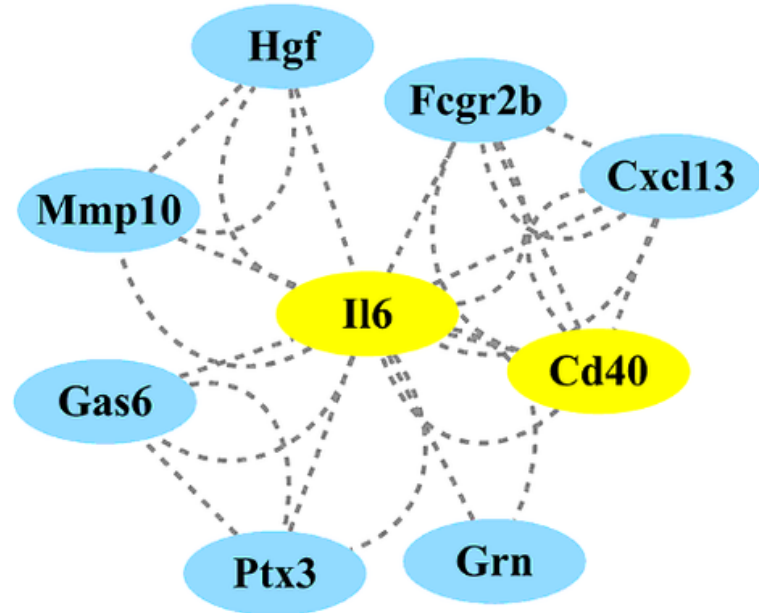

CD40

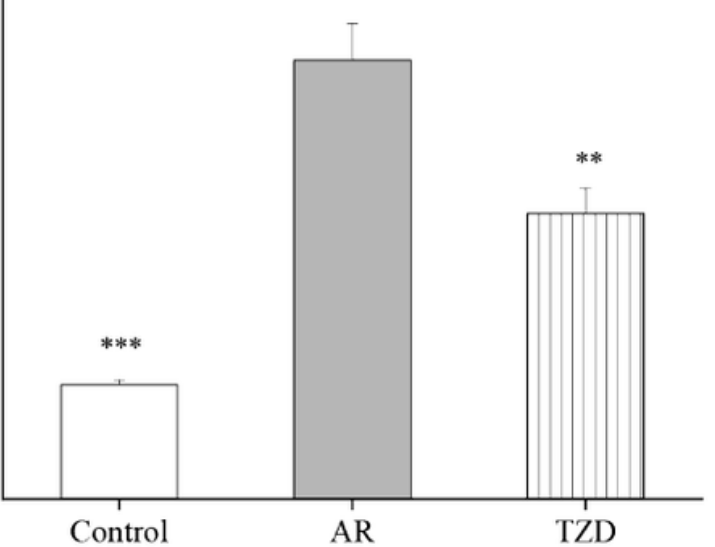

Figure 7

Selection of key DEPs. (A) Venn diagrams showing the distribution of DEPs between the model and TZD groups and the model and control groups. (B) The PPI network of DEGs and four hub-proteins were obtained by MCODE and the MCC score, which is represented by a yellow color. (C) Hub-protein levels of IL-6 and CD40. Proteomics of shared DEPs among the control group (Control), the AR group (AR) and the TZD group (TZD). The values represent the mean \pm SE. Significant differences at ${ }^{\star *} P<0.01$, ${ }^{\star * P}<0.001$, compared with the AR group. 
<smiles>O=C(Cl)C(=O)c1ccccc1</smiles><smiles>COc1c(O)cc(O)c2c(=O)cc(-c3ccccc3)oc12</smiles>

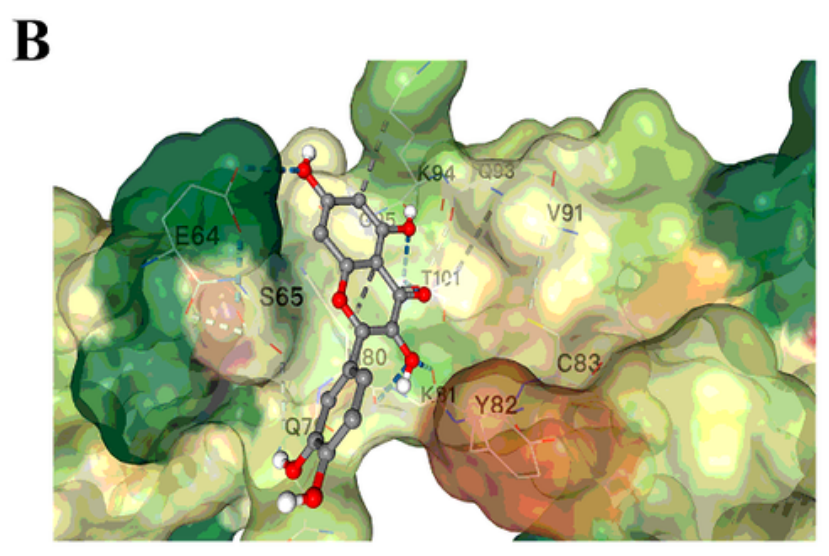

CD40-Quercetin

D

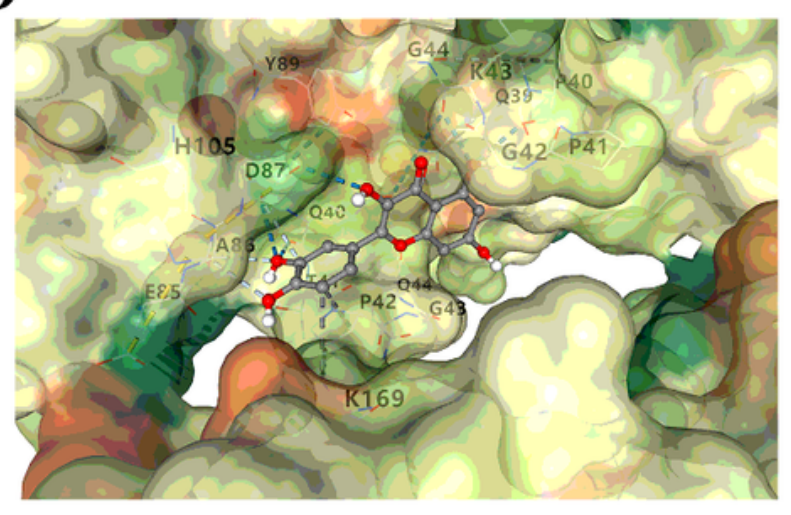

IL6-Quercetin

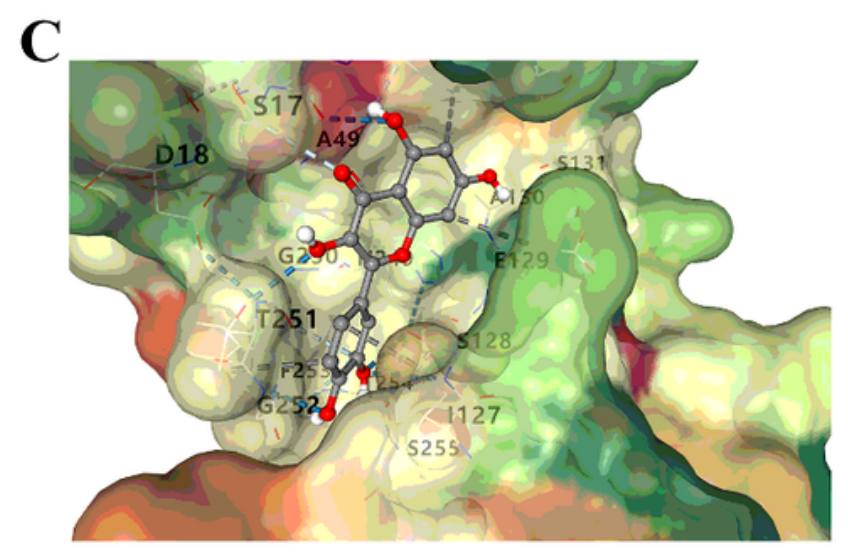

\section{CD40L-Quercetin}

$\mathbf{E}$

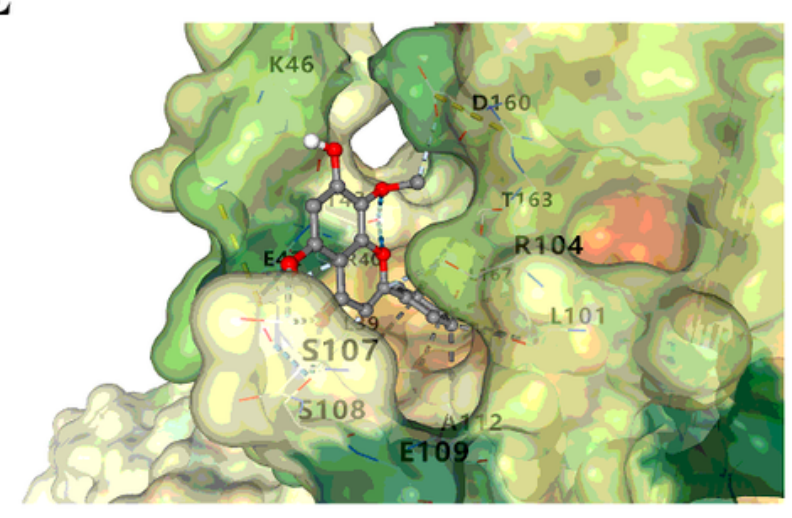

IL6-Wogonin

Figure 8

Action modes of active compounds with key targets. A Compound structure of quercetin and wogonin; B CD40-Quercetin; C CD40L-Quercetin; D IL-6-Quercetin; E IL6-Wogonin; 